\title{
Ein Rückblick auf die internationale Konferenz „Phrasenstrukturen und interpretationen im Gebrauch“ aus der Reihe „Linguistische Treffen in Wroclaw", 25-27. September 2014
}

Die Tagungen aus dem Zyklus „Linguistische Treffen in Wrocław“ werden im zweijährigen Rhythmus am Institut für Germanische Philologie der Universität Wrocław veranstaltet. Die Tradition, jedes zweite Jahr ein internationales Treffen der Wissenschaftler aus dem Bereich der Linguistik zu organisieren, wurde 2006 mit der Konferenz „Linguistik-Tage in Wrocław“ eingeleitet, die in Zusammenarbeit mit der Gesellschaft für Sprache und Sprachen organisiert wurde. 2008 und 2010 fanden ebenfalls sprachwissenschaftliche Fachtagungen in Wrocław statt, deren Schwerpunkt jeweils die germanistische, aus unterschiedlichen Perspektiven betrachtete Linguistik bildete. Zwei Jahre später wurde mit der Wahl des Themas der Konferenz ein breiteres Projekt begonnen, im Rahmen dessen unterschiedliche Ebenen des Sprachsystems wissenschaftlich (neu) ergründet werden sollten. Demnach wurde als Hauptthema der Tagung im Jahre 2012 ,das Wort‘ im weiteren Sinne festgelegt. Im Mittelpunkt der diesjährigen Konferenz, die vom 25. bis zum 27. September 2014 in Wrocław stattfand, stand ,die Phrase‘. Der Einladung zur Konferenz lässt sich Folgendes entnehmen:

Unsere Absicht ist es, im Rahmen der Konferenz verschiedene Themen zu berühren, die die breit begriffenen Phrasen, ihre Art, Zuordnung, Zusammensetzung, Funktionen, Wirkung, Verwendungsbereiche und -arten, das Kategorisieren, Typologisieren, ihre Kontexte, Interpretationen, Deutungs-, Interpretations- und Perzeptionsmöglichkeiten etc. ansprechen und das in allen Bereichen der linguistischen Forschung. Möglicherweise auch interdisziplinär. ${ }^{1}$

1 http://www.ifg.uni.wroc.pl/stacjonarne/konf/ltinwroclaw.html (25.12.2014.). 
Das der Einladung entnommene Zitat zeigt das breite Spektrum der möglichen linguistischen Assoziationen mit dem Thema der Phrase.

Die Tagung wurde von dem Lehrstuhl für Deutsche Sprache und dem Lehrstuhl für Angewandte Linguistik konzipiert und organisiert. An der Veranstaltung und der erfolgreichen Durchführung der Konferenz wirkten Mitarbeiter, Doktoranden und Studenten der Germanischen Philologie der Universität Wrocław mit. Die Konferenz wurde in Zusammenarbeit mit der Universität Leipzig sowie unter der Schirmherrschaft der Stiftung für deutsch-polnische Zusammenarbeit und des Verlags C.H. Beck veranstaltet.

An der Konferenz nahmen 87 Linguistik-Interessierte teil, die aus verschiedenen Ländern Europas stammen, z. B. aus Deutschland, Frankreich, Spanien, Russland, Tschechien, Ungarn. Die Mehrheit der Teilnehmer waren Linguisten aus Polen, wobei hier auch Vertreter bedeutender polnischer germanistischer Institute anwesend waren, z. B. Kraków, Warszawa, Poznań, Łódź, Rzeszów, Bydgoszcz, Olsztyn, Częstochowa u a.

Das Themenspektrum der Konferenz erstreckte sich, wie schon angedeutet, über die ganze Bandbreite der Themen und Fragen, die mit der Phrase verbunden sind. Dennoch ließen sich einerseits bestimmte Punkte der Tagung hervorheben, die für die Organisatoren in diesem Jahr von besonderer Bedeutung waren, und andererseits thematische Schwerpunkte ausmachen, nach denen die Arbeit in inhaltlich abgestimmte Sektionen gegliedert werden konnte. In diesem Jahr ging der offiziellen Eröffnung der Konferenz und dem eigentlichen Vortragsteil ein Blockseminar zur Phraseologie voran, das an Studenten und alle interessierten Gäste gerichtet war. Dr. habil. Petra Szatmári (Budapest) führte die Teilnehmenden in das Thema der Modifikation von Phraseologismen und in die Entstehung der phraseologischen Sprachspielereien ein. Vor dem theoretischen Hintergrund zum Thema der beiden Phänomene wurden Fallbeispiele vorgestellt und diskutiert. Die zweite Leiterin des phraseologischen Blockseminars, Dr. habil. Hana Bergerová (Ústi nad Labem), beschäftigte sich in ihrem Teil des Seminars mit Phraseologismen aus didaktisch-orientierter Perspektive. Ferner handelte es sich um solche Fragen, welche Methoden man einsetzen kann, um die Phraseologismen im DaF-Unterricht erfolgreich vermitteln zu können, sowie welche Materialien aus welchen Quellen man im Unterricht einsetzen und welche Übungstypen man auswählen kann, um den Unterricht interessanter und informativer zu gestalten. Das Blockseminar erfreute sich unter den Germanistikstudenten und Konferenzteilnehmern eines großen Interesses. Es bot einen 
guten Ausgangpunkt für die Konferenz und die erste Möglichkeit der Kontaktaufnahme unter Wissenschaftlern und Studenten.

Direkt nach dem phraseologischen Blockseminar wurde die Konferenz von dem Dekan der Philologischen Fakultät der Universität Wrocław, Prof. Dr. Marcin Cieński, der Direktorin des Instituts für Germanische Philologie der Universität Wrocław, Prof. Dr. Iwona Bartoszewicz und von Prof. Dr. Danuta Rytel-Schwarz von dem Institut für Slavistik der Universität Leipzig offiziell eröffnet. Der feierlichen Eröffnung der Tagung folgten Plenarvorträge, in denen das Werk und das Schaffen des 2013 verstorbenen Prof. Dr. Eugeniusz Tomiczek, des langjährigen Direktors des Instituts für Germanische Philologie der Universität Wrocław, gewürdigt wurden. Im Block der Plenarvorträge hielt Prof. Dr. Marek Hałub (Wrocław) den Eröffnungsvortrag, in dem er die langjährige Tätigkeit von Prof. Dr. Eugeniusz Tomiczek am Institut für Germanische Philologie schilderte. Nachfolgend präsentierte Prof. Dr. Iwona Bartoszewicz (Wrocław) das Porträt von Prof. Dr. Eugeniusz Tomiczek als Forscher und akademischen Lehrer. Die drei weiteren Referate wurden zwar nicht direkt der wissenschaftlichen Tätigkeit von Prof. Dr. Eugeniusz Tomiczek gewidmet, sie knüpften jedoch mit ihren Themen an seinen Interessenbereich an. In diesem Zusammenhang befasste sich Prof. Dr. Zofia Berdychowska (Kraków) mit personaldeiktischen Phrasenstrukturen als Referenzmittel, genauer gesagt mit ihrem Aufbau, ihren Beschränkungen im Hinblick auf ihre Struktur sowie mit ihren Funktionen. Silvia Bonacchi (Warszawa) ging in ihrem Beitrag auf die (Un)Höflichkeitsforschung ein, wobei sie das Thema interkulturell auffasste und den Fokus auf mögliche Forschungsperspektiven legte. Alina Jurasz (Wrocław) und Danuta Rytel-Schwarz (Leipzig) griffen das Thema der Anrede- und Schlussformeln im universitären Bereich anhand der eigenen Korrespondenz mit polnischen und deutschen Studierenden auf. Mit diesem Referat wurde der erste Tag der Konferenz abgeschlossen, der der Ehrung des Werkes und der didaktischen und organisatorischen Tätigkeit des verdienten Professors Eugeniusz Tomiczek gewidmet wurde.

Die zwei weiteren Tage der Tagung wurden nach gleichem Muster strukturiert $^{2}$. Sie wurden jeweils mit zwei Plenarvorträgen eröffnet, denen die Arbeit in Sektionen folgte. Den ersten Plenarvortrag hielt Michael Thiele (Karlsruhe/ Frankfurt am Main), der sich mit Phrasen mit der Komponente Herz in Hermann

2 Der vorliegende Bericht ist nicht nach dem chronologischen, sondern nach dem thematischen Prinzip aufgebaut. 
August Franckes Predigten beschäftigte. Martine Dalmas (Paris) thematisierte im nachfolgenden Plenarvortrag anaphorische attributive Adjektivphrasen im Lichte der kognitiven Linguistik mit besonderer Berücksichtigung ihrer Brückenbildungsfunktion. Am abschließenden Konferenztag hielt Michał Kotin (Zielona Góra) den eröffnenden Vortrag, in dem er sich zum Thema der Phraseologismenbildung äußerte. Im Vortrag von Georg Schuppener (Leipzig) wurde die rechtsextreme Phraseologie am Beispiel der Begrüßungen und Schlussformeln, die rechtsextremen Internetseiten entnommen wurden, besprochen.

Innerhalb der zwei Konferenztage ließen sich die Referate in neun Sektionen einordnen, wobei jeweils in vier Sektionen, zwischen denen man wechseln konnte, parallel vorgetragen und diskutiert wurde. Im weiteren Programm konnten somit die folgenden thematischen Blöcke unterschieden werden:

- Phraseologismus: Definition und Komponentenbestand,

- Phraseologismen im Gebrauch,

- Kollokationen und Funktionsverbgefüge,

- Phrasen: Definition und Komponentenbestand,

- Phrasen im Gebrauch,

- Phrasen kontrastiv,

- Phrasen als Objekt didaktische Reflexion,

- Zwischen Phrasen und Merksatz: normative, methodische und didaktische Aspekte der Wissenschaftssprache,

- Sprichwörter/Phrasen als Mikrotexte.

In der ersten Sektion Phraseologismus: Definition und Komponentenbestand wurde ein Referat gehalten. Joanna Szczęk und Marcelina Kałasznik (Wrocław) beschäftigten sich in ihrem Referat mit deutschen Phraseologismen - Trophotismen, die in ihrem Komponentenbestand Bezeichnungen für Kulinarien enthalten.

Die zweite Sektion Phraseologismen im Gebrauch war umfangreich. Im Referat von Reinhold Utri (Warszawa) wurde die Plurizentrik der deutschen Sprache anhand der phraseologischen Einheiten im Österreichischen Deutschen thematisiert. Petra Szatmári (Budapest) ging in ihrem Referat auf das Phänomen der Verknüpfung phraseologischer Einheiten zu komplexen Phrasen mit überdurchschnittlich großer Informativität und Expressivität ein. Małgorzata Sieradzka (Rzeszów) stellte in ihrem Vortrag die Analyse der im Roman Wojna polska-ruska auftretenden modifizierten Phraseologismen und die Strategien deren Übersetzung ins Deutsche vor. Magdalen Lisiecka-Czop (Szczecin) setzte 
sich zum Ziel, die Erfassung der maritimen fachsprachlichen Phraseologismen sowohl in fachsprachlichen Lexika als auch in Universalwörterbüchern der deutschen Gegenwartssprache unter die Lupe zu nehmen. Janusz Pociask (Bydgoszcz) beleuchtete die Funktion und den Status der phraseologischen Ausdrücke in Titeln der wissenschaftlichen Beiträge. Jiřina Malá (Brno) überprüfte in dem Referat deutsche Filmrezensionen auf die Verwendung von abgegriffenen Phrasen und modifiziert verwendeten Phraseologismen hin. Małgorzata Czarnecka (Wrocław) befasste sich mit stabilen Phrasen aus der Zeit der Volksrepublik Polen und deren Verankerung in der Erinnerung der Polen. Dominika Janus (Gdańsk) stellte in ihrem Vortrag die Analyse von Phrasen vor, die deutschen und polnischen Todesanzeigen entnommen wurden, und dies mit dem Ziel, ihre Frequenz, Funktionen und Perzeptionsmöglichkeiten zu erforschen. Anna Gondek und Joanna Szczęk (Wrocław) gingen in ihrem Referat dem Bild des Teufels in der deutschen Sprache nach. Hanna Kaczmarek (Częstochowa) leistete mit ihrem Vortrag einen Beitrag zur Phraseographie und Phraseodidaktik, indem sie in den Phraseologismen versprachlichte Konzepte der ,Freude“ untersuchte und ihre lexikographische Erfassung unter die Lupe nahm.

In der Sektion Kollokationen und Funktionsverbgefüge wurden fünf Vorträge gehalten. Anna Radzik (Kraków) beschäftigte sich mit verbonominalen Konstruktionen und ihrer Festigkeit. Bogusława Rolek (Rzeszów) stellte in ihrem Referat Überlegungen über den Gebrauch sowie das semantische und syntaktische Potenzial der konventionalisierten Wortverbindungen in der Unternehmenskommunikation an. Kornelia Kołupajło (Poznań) setzte sich in ihrem Vortrag mit dem Terminus der Kollokation in der deutschen und polnischen Forschungsliteratur auseinander. Agnieszka Stawikowska-Marcinkowska (Łódź) ging der Frage der Wortgruppen aus dem Bereich der Börsensprache nach. Marcelina Kałasznik (Wrocław) befasste sich in ihrem Vortrag mit adjektivischen Kollokationen mit folgenden Komponenten: Star, Promi und Idol.

In der Sektion Phrasen: Definition und Komponentenbestand wurden acht Referate gehalten. Artur Tworek (Wrocław) machte die phonetische Ebene oberhalb der Sprachlaute zum Gegenstand seines Referats. Anna Pieczyńska-Sulik (Poznań) untersuchte in ihrem Referat die Ikonizität der Sprache auf der Ebene der Phrase. Agnieszka Poźlewicz (Poznań) setzte sich zum Ziel, das Phänomen und die Mittel der sprachlichen Hervorhebung anhand von unterschiedlichen Textsorten zu erforschen. Daniela Elsner (Bochum) befasste sich in ihrem Referat mit der Form und Funktion von Konstruktionen im Nachfeld. Jarosław 
Aptacy (Poznań) thematisierte in seiner Präsentation Sätze mit Verben, die Akkusativobjekt regieren, und ihre Negation. Edyta Błachut (Wrocław) stellte drei Schemata des Tautologisierens vor und versuchte anhand der Analyse der Einzelbeispiele, die sich jeweils einem der Muster zuordnen ließen, zu beweisen, dass sie informativ und sinnvoll sind. Przemysław Staniewski (Wrocław) setzte sich in seinem Beitrag mit dem Phänomen der sprachlichen Synästhesie und ihren Typen auseinander. Marek Biszczanik (Zielona Góra) zeigte in seinem Vortrag die fehlende Übereinstimmung der einzelnen Pragmeme eines Lexems am Beispiel des Lexems ,zu` bei seiner Desemantisierung und beim Phrasenbildungsvorgang.

In der Sektion Phrasen im Gebrauch wurden 17 Referate gehalten. Anna Dargiewicz (Olsztyn) nahm Phrasen insbesondere englischer Herkunft als Bestandteile der sog. substantivischen Hybridkomposita unter die Lupe. Marcin Maciejewski (Poznań) ging in seinem Referat der Funktion und dem propositionalen Gehalt solcher Phrasen wie Ich bin katholisch/evangelisch nach. Sebastian Kiraga (Regensburg) beschäftigte sich in seinem Referat mit Äußerungen aus katholischen Messen, die unterschiedlich interpretiert werden können und versuchte mit Einbeziehung sowohl linguistischer als auch theologischer Kriterien, eine eindeutigere Auslegung der untersuchten Phrasen zu liefern. Elżbieta Pawlikowska-Asendrych (Częstochowa) legte in ihrem Beitrag die Bedeutung und die Verbindbarkeit der Nominalphrase Erinnerung dar. Věra Höppnerová (Prag) analysierte in ihrem Vortrag Phrasen, Kollokationen und Ausdrücke, die für die wirtschaftliche Korrespondenz typisch sind. Grażyna Strzelecka (Warszawa) machte häufig gebrauchte Phrasen aus der Wirtschaftspresse Ende des 19. und 20. Jahrhunderts zum Gegenstand ihres Referats. Tomasz Jaśków (Görlitz) setzte sich zum Ziel, Entschuldigungen von Politikern genauer zu erforschen und sie aus sprachwissenschaftlicher Perspektive zu betrachten. Magdalena Kolbusz (Rzeszów) befasste sich mit unterschiedlich aufgebauten Nominalphrasen und ihrer Verwendung in fachlichen und populärwissenschaftlichen Texten aus der Zeitschrift „Höhle“. Evgeniya Kakzanova (Moskau) stellte das Gedicht „Verwerfung. Bedeutung. Permutation“ ins Zentrum ihrer Analyse und untersuchte seine einzelnen Phrasen im Hinblick auf ihre Struktur und Semantik. Jarosław Ślawski (Kraków) behandelte die spezifischen Merkmale der Sprache von Erich Kästner anhand des Kinderromans Emil und die Detektive mit besonderer Berücksichtigung der Phraseologie und ihrer Bedeutung für die Erweiterung des Wortschatzes des kindlichen Lesers. Joanna Pędzisz (Lublin) stellte eine Analyse der Inter- 
netkommentare im deutschen Diskurs über Windparks dar. Roman Opiłowski (Wrocław) investigierte Beziehungen zwischen Phrasemen aus Überschriften und Bildern in deutschen und polnischen Zeitungstexten und unternahm einen Versuch, sie zu kategorisieren. Joanna Stroisz (Rzeszów) ergründete die Bedeutung der Phraseologismen in der Wirtschaftssprache. Richard Rothenhagen und Marek Halo (Brno) präsentierten den neu erschienenen Band des Atlas der deutschen Mundarten in Tschechien und gingen auf die Schwierigkeiten bei dessen Entstehung ein. Jan Hajduk (Kielce) skizzierte das sprachliche Bild des Weges anhand von lexikographischen Definitionen und der Verwendung des Wortes in Grabinschriften. Katrin Ankenbrand (Wrocław) ging in erster Linie der Frage nach, welche sprachlichen Mittel im Sprechakt des Dankens gebraucht werden können, damit der Dank überzeugend wirkt. Nikolai Czemplik (Leipzig) untersuchte Phrasenstrukturen in ausgewählten deutschen Dialekten.

In der Sektion Phrasen kontrastiv wurden zehn Referate gehalten. Janusz Taborek (Poznań) behandelte die Erfassung der mehrgliedrigen Einheiten in einem neuerschienenen polnisch-russisch-englisch-deutschsprachigen Wörterbuch der Fußballsprache. Łukasz Plęs (Łódź) beschäftigte sich in seinem Beitrag mit den Strategien der Übersetzung von polnischen Partizipialphrasen ins Deutsche am Beispiel der Prosatexte. Paweł Bąk (Rzeszów) präsentierte den Beitrag Zur Zweisprachigkeit der öffentlichen Diskurse. Andrzej Szubert (Poznań) konzentrierte sich in seinem Referat auf eine ausgewählte Gruppe von dänischen phraseologischen Einheiten, die in ihrem Komponentenbestand das Substantiv tid enthalten, sowie auf ihre Äquivalente im Deutschen. Renata Nadobnik (Gorzów Wielkopolski) bezog sich in ihrem Vortrag auf die lexikographische Erfassung der Phraseologismen, die im Sprachenpaar Deutsch-Polnisch im Spiegelbild auftreten, und analysierte sie in Bezug auf ihre Struktur und Bildhaftigkeit hin. Mariusz Frąckowiak (Opole) thematisierte Phraseologismen sowie ihre Strukturen und Modifikationen in der aktuellen deutschen Presse. Stefan Ludwin (Jelenia Góra) machte Die Adjektive auf-bar, -lich und-fähig als PassivParaphrasen und ihre polnischen Entsprechungen zum Thema seines Referats. Michał Smułczyński (Wrocław) unternahm einen Versuch, die Auffassungen der Direktiv- und Situativergänzung zu revidieren. Mateusz Sajna (Wrocław) erörterte das Phänomen der Amalgame am Beispiel der Eigennamen aus Filmen und Video-Spielen. Rafał Jakiel (Wrocław) stellte Eigennamen in vielerlei Hinsicht in den Mittelpunkt seines Beitrags. 
In der Sektion Phrasen als Objekt didaktischer Reflexion wurden sechs Referate gehalten. Janusz Sikorski (Warszawa) stellte in seinem Referat ein Konzept der Einbeziehung der Phraseologie in die korrektive Phonetik des Deutschen vor. Grit Mehlhorn (Leipzig) befasste sich in ihrem Referat mit Intonationsphrasen zweisprachig aufgewachsener Kinder und ihrer Eltern. Maria Yastrebova (Leipzig) stellte eine Analyse vorgefertigter Phrasen in Wegbeschreibungen bilingualer Kinder vor. Joanna Targońska (Olsztyn) stellte in ihrem Referat die Frage, welchen Kollokationsbegriff die Fremdsprachendidaktik braucht. Małgorzata Niemiec-Knaś (Częstochowa) befasste sich in ihrem Beitrag mit der Interaktion von Emotion und Kognition sowie mit ihrer Bedeutung und ihrem Nutzen für den DaF-Unterricht. Beata Rusek (Częstochowa) stellte im Referat Reflexionen darüber an, wie man mit Chunks freies Sprechen im DaF-Unterricht fördern kann.

In der Sektion Zwischen Phrasen und Merksatz: normative, methodische und didaktische Aspekte der Wissenschaftssprache hielt Karin Pittner (Bochum) das erste Referat, in dem sie das Phänomen der Subjektschübe, seine Formen und Funktionen in wissenschaftlichen Texten untersuchte. Agnieszka Nyenhuis (Bochum) befasste sich mit dem Begriff der Interpersonalität in wissenschaftlichen Texten. Tadeusz Zuchewicz (Zielona Góra) betonte in seinem Vortrag die Rolle des richtigen Definierens in wissenschaftlichen Abhandlungen, erläuterte Anforderungen an Definitionen sowie unterschiedliche Definitionsweisen. Michał Kotin (Zielona Góra) befasste sich mit intern und extern bedingten Differenzen zwischen verschiedenen Standards des Verfassens von wissenschaftlichen Arbeiten. Magdalena Plinta (Wrocław) stellte in ihrem Referat Ergebnisse einer unter chinesischen Studenten durchgeführten Umfrage bezüglich des Verfassens von wissenschaftlichen Texten. Marta Rogozińska (Wrocław) thematisierte in ihrem Vortrag Elemente, die Phrasen einleiten, wobei sie sich auf die gesprochene Fachsprache der Wirtschaft konzentrierte. Lesław Cirko (Wrocław) stellte in seinem Referat Überlegungen über Verbalphrasen in deutschen und polnischen Texten aus dem Bereich der Philologie an. Adam Gołębiowski (Wrocław) beschäftigte sich mit der Prädizierung in deutschen und polnischen Texten mit besonderer Berücksichtigung der wissenschaftlichen Texte. Sandra Seidel (Wrocław) setzte sich zum Ziel, den Terminus des Standards näher zu betrachten und ihn im Hinblick auf bedeutungsähnliche Begriffe zu situieren. 
In der neunten Sektion Sprichwörter/Phrasen als Mikrotexte präsentierte Janusz Stopyra (Wrocław) dänische Versionen von Sprichwörtern, die im Polnischen und im Deutschen gebräuchlich sind. Józef Jarosz (Wrocław) griff in seinem Referat die Frage des Einsatzes von Phraseologismen und Sprichwörtern in Grabinschriften des 19. und 20. Jahrhunderts auf. Elżbieta Kucharska-Dreiß (Wrocław) stellte eine Auswertung der deutschsprachigen Stammbuchsprüche des 16. und 17. Jahrhunderts aus dem Stechow's Stammbuchsprüche-Schlüssel dar.

Anhand des kurzen Umrisses der Themen, die auf der Konferenz berührt wurden, kann festgestellt werden, dass das inhaltliche Spektrum ziemlich breit war, wobei dies von allen als positiv empfunden wurde. Die internationale Dimension der Konferenz ermöglichte es den Gästen, sich mit den in anderen Ländern aktuellen Forschungsrichtungen und -methoden im Bereich der germanistischen Linguistik vertrauter zu machen. Die Tagung bot auch, wie immer, eine gute Möglichkeit zum internationalen wissenschaftlichen Austausch, an dem sich sowohl Studenten, Doktoranden und teilnehmende Wissenschaftler rege beteiligten. Es bleibt nur zu hoffen, dass die für das Jahr 2017 angesagte 6. Tagung aus dem Zyklus „Linguistische Treffen in Wrocław“ sich ebenfalls einer großen Resonanz erfreuen wird. 